\title{
A New Extended-Gamma Family of Distributions: Properties and Applications
}

\author{
Bachioua Lahcene \\ Department of Basic Sciences Prep. Year, P.O. Box 2440, University of Hail, Hail, Saudi Arabia.
}

\begin{abstract}
How to cite this paper: Bachioua Lahcene. (2021) A New Extended-Gamma Family of Distributions: Properties and Applications. Journal of Applied Mathematics and Computation, 5(1), 9-17.

DOI: $10.26855 /$ jamc.2021.03.002
\end{abstract}

Received: November 30, 2020

Accepted: December 29, 2020

Published: January 20, 2021

*Corresponding author: Bachioua Lahcene, Department of Basic Sciences Prep. Year, P.O. Box 2440, University of Hail, Hail, Saudi Arabia.

Email: drbachioua@gmail.com

\begin{abstract}
In this paper, the author proposes a seven-parameter lifetime model called extended generalization of the gamma distribution, which stems from the expansion of the gamma function. The expanded generalization of both function generalization and gamma distribution has been highlighted in recent decades for their widespread use in important application areas in pure and mixture models, and this expansion provides a comprehensive mathematical treatment to trace the behavior of some random phenomena represented by this distribution. The author introduces some key characteristics of extended generalization to gamma, modified and extended gamma, and provides moments, quantitative function, and other important metrics. The findings of this work will be useful and constructive for practitioners in various fields of theoretical and applied sciences.
\end{abstract}

\section{Keywords}

Moment Generating Function, Mean Deviation, Generalization Gamma Distribution, Reliability, Generating and Quantile Functions, Hazard Rate Function, Parameter Estimation, Estimation Methods

\section{Introduction}

The gamma distribution is the most popular model for analysing skewed data and hydrological processes. Standard lifetime distributions usually present very strong restrictions to produce bathtub curves, and thus appear to be inappropriate for analysing data with this characteristic. The three-parameter generalized gamma distribution [3] includes as special models the exponential, Weibull, gamma and Rayleigh distributions, among others [1, 2, 3].

One of the important families of distributions in lifetime tests is the extended gamma distribution. The extended generalized gamma distribution is a highly known distribution due to its utility in modelling lifetime data where the hazard rate function is monotone in special cases $[4,5]$.

In 2004, Bachioua proposed an extended gamma distribution with the special feature of being able to adapt failure rates with bathtub and unimodal shape curves [6].

Extended Generalized gamma distribution has proved to be of considerable interest in the field of reliability. It is a reasonable model for life-time distribution of a component (or a system of components) [7, 8]. This distribution does not have the expected impact due to its complicated form. Among these, the extended five gamma distribution was presented by Bachioua et al., among others. A good review of these models is presented in Abdul Moiz and Bachioua [8].

Due to the importance of the generalized gamma function, its basic properties have been studied by Bachioua [9, 10]. Because of the incomplete gamma function, we remember that the first to do this diligence in the research was Led Legendre (1811) as decompose the gamma function into the incomplete gamma functions, and which represent. The upper incomplete gamma function is of the form: 


$$
\Gamma(\alpha, x)=\int_{x}^{+\infty} t^{\alpha-1} e^{-t} d t
$$

Whereas the lower incomplete gamma function defined as:

$$
\gamma(\alpha, x)=\int_{0}^{x} t^{\alpha-1} e^{-t} d t
$$

Since the ordinary gamma function defined as

$$
\Gamma(\alpha)=\gamma(\alpha, x)+\Gamma(\alpha, x)
$$

Chaudhry and Zubair modified the domain of these functions by inserting a regularization factor $\exp (-b / t)$ in the form equation (2). We note the following relation equation by [9]:

$$
\Gamma_{b}(\alpha)=\int_{0}^{+\infty} t^{\alpha-1} e^{\left(-t-\frac{b}{t}\right)} d t
$$

The function formula can be returned in two parts by the upper incomplete gamma function and the lower incomplete gamma function by:

$$
\Gamma_{b}(\alpha)=\gamma_{b}(\alpha, x)+\Gamma_{b}(\alpha, x)
$$

Due to the huge success of the gamma function, many authors have defined and discussed different types of gamma function in recent years. Recently, Kobayashi (1991) considered a generalized gamma function in the generalized form by [1];

$$
\Gamma_{r}(\alpha, n)=\int_{0}^{+\infty} t^{\alpha-1}[t+n]^{-r} e^{-t} d t
$$

Kobayashi considered plane wave diffraction by strip, using the Weiner-Hopf technique [11]. Bachioua [6] generalized Kobayashi gamma function by introducing for the first time a parameter $\mathrm{m}$ and representing the new gamma function by [5]:

$$
\Gamma_{r}(\alpha, n, m)=\int_{0}^{+\infty} t^{\alpha-1}\left[t^{m}+n\right]^{-r} e^{-t} d t
$$

Bachioua and Abdul Moiz [7] defined and studied an extended generalized gamma function [12].

\section{Mathematical Description and Theory}

In this section, after going through the contents of the articles, and the most important modifications proposed by many researchers and authors, it is required to make a research to rephrase the extended roughness. A new extended generalized gamma function is defined involving a parameter function, through the following proposed model:

$$
\Lambda_{r}(\alpha, n, m, p, \lambda, b)=\int_{0}^{+\infty} t^{\alpha-1}\left[t^{m}+n\right]^{-r} e^{-\lambda t^{p-1}-\frac{b}{t}} d t
$$

It is important and useful to look for other integral representations of the extended gamma function and extended incomplete gamma function both as a check that the extension is natural, simple, and useful for late use. For the broad investment and maximum benefit of the proposed model, the researchers also propose a proposal for the extended function of the new incomplete gamma function as follows:

$$
\Lambda_{r}(\alpha, n, m, p, \lambda, b)=\gamma_{r}(\alpha, n, m, p, \lambda, b: x)+\Lambda_{b}(\alpha, n, m, p, \lambda, b: x)
$$

The proposed new function of the extended incomplete gamma function is given as follows:

$$
\left\{\begin{array}{l}
\gamma_{r}(\alpha, n, m, p, \lambda, b: x)=\int_{0}^{x} t^{\alpha-1}\left[t^{m}+n\right]^{-r} e^{-\lambda t^{p-1}-\frac{b}{t}} d t \\
\Lambda_{r}(\alpha, n, m, p, \lambda, b ; x)=\int_{x}^{+\infty} t^{\alpha-1}\left[t^{m}+n\right]^{-r} e^{-\lambda t^{p-1}-\frac{b}{t}} d t
\end{array}\right.
$$

It is also useful to look for relationships between the original beta function and its extension. In this connection, we first provide a relationship between them. With ease and simplicity, the circular reviewed by the researcher, Chaudhry et al in $[9,13]$ can be expressed as follows:

$$
\gamma_{r}(\alpha, n, 1,2,1, b: x)=\int_{0}^{x} t^{\alpha-1}[t+n]^{-r} e^{-t-\frac{b}{t}} d \gamma_{b}(\alpha, n: x)
$$

\section{Proposed Model}

The early generalization of gamma distribution can be traced back to Amoroso who discussed the generalized gamma distribution and applied it to fit income rates [14]. Johnson et al. [15] proposed a four- parameter generalized gamma 
distribution, which reduces to the generalized gamma distribution with three parameters defined by Stacy [3] when the location parameter is set to zero. Agarwal and Al-Saleh used generalized gamma distribution to study hazard rate [1]. They derived another generalization of Stacy's generalized gamma distribution using exponentiated method, and applied it to lifetime and survival analysis.

The extended generalized gamma distribution, which was introduced by Bachioua [6] presents a flexible family with varying location, scale, shapes and hazard functions often suitable for modeling survival data. Of late, Jones [16] employed quantile functions to generate T-X family of distributions. For assessment of methods for generating distributions see Jones [16].

In this work, we define a new extension of the generalized gamma distribution. The new generalizations of the gamma distribution, and the extended gamma distribution were introduced by Abdul Moiz and Bachioua [7]. The new modified generalized gamma distribution, called the extended generalized gamma distribution has been introduced to provide greater flexibility in modeling data from a practical viewpoint [12]. A continuous random variable $\mathrm{X}$ is said to have a new extended generalized gamma distribution with seven parameters named NEGGD(7) if its probability density function (p.d.f.) is expressed as:

$$
f_{r}(x ; \alpha, n, m, p, \lambda, b)=\frac{x^{\alpha-1}\left[x^{m}+n\right]^{-r} e^{-\lambda x^{p-1}-\frac{b}{x}}}{\Lambda_{r}(\alpha, n, m, p, \lambda, b)}
$$

where $\alpha, n, m, p, \lambda, b$ are positive real numbers which represent scale numbers while the shape parameter, $r$, is a real number and $\Lambda_{r}(\alpha, n, m, p, \lambda, b)$ presents the extended generalized gamma function. Many commonly used probability distributions are special cases of the extended generalized gamma distribution. The graph of its pdf and hazard function for various values of the parameters are showed in Figure 1 and Figure 2.

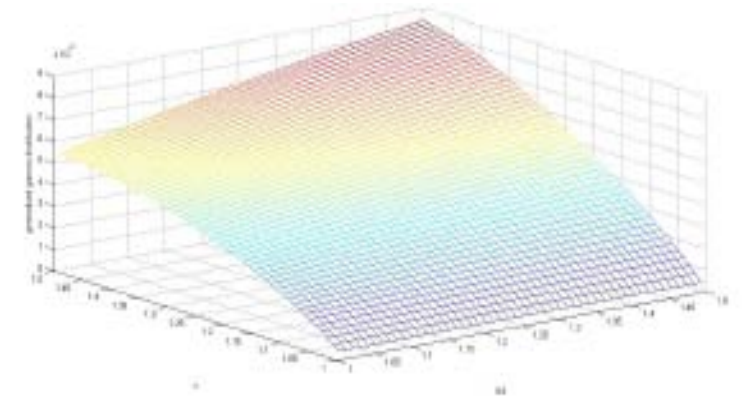

Fig. 1. graph of pdf function versus $\alpha$ with $r=1.3 ; \lambda=1.2 ; n=0.9 ; m=1 ; p=0.2 ; b=0.7$.

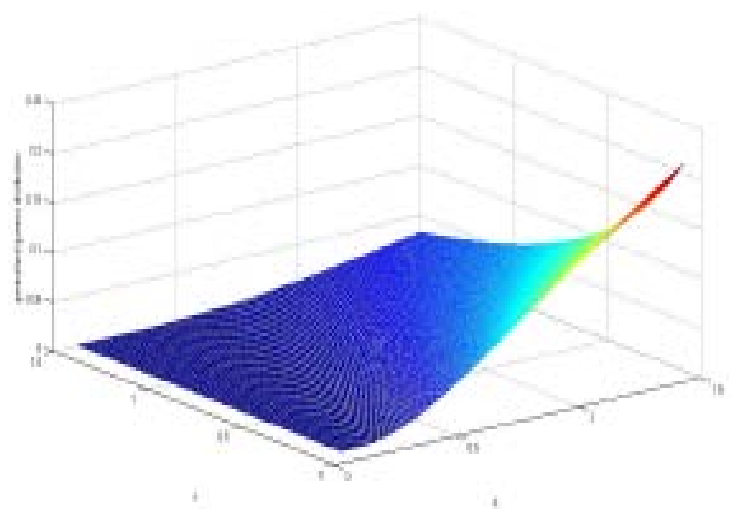

Fig. 2. graph of pdf function vernus $\lambda$ with $r=0.2 ; \alpha=1.5 ; n=0.5 ; m=0.3 ; p=0.1 ; b=1$.

Figure $1 \& 2$. PDF for different values of $r$ and $\alpha, n, m, p, \lambda, b$, giving a clear idea of the changes caused by the different values of these parameters, those interested can study special cases and note the distinct cases in each change. 
It can have a constant, decreasing, increasing, upside-down bathtub or bathtub-shaped hazard rate function depending on the parameter values. We demonstrate that the new density function can be expressed as a mixture of generalized gamma densities. We illustrate the flexibility of the new distribution by means of two applications to real datasets.

We provide some new mathematical properties of the transmuted generalized gamma distribution defined from the family pioneered by Bachioua. The graph of its pdf and hazard function for various values of the parameters are showed in Figures 3, 4, 5.

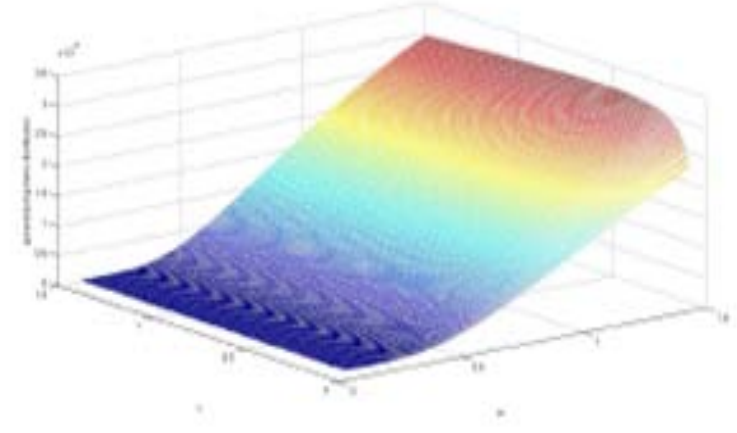

7ig. 3. graph of pdf function venuas $m$ with $r=0.3 ; a=1.01 ; n=0.4 ; \lambda=0.7 ; p=0.01 ; b=1.4$.

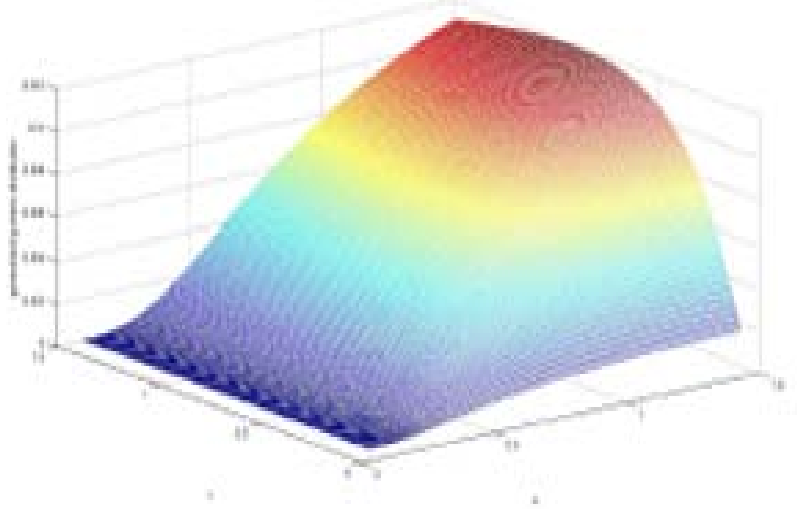

Fig. 4. graph of pdf function venus $n$ with $r=1 ; a=0.3 ; m=1.2 ; \lambda=0.7 ; p=0.6 ; b=1$.

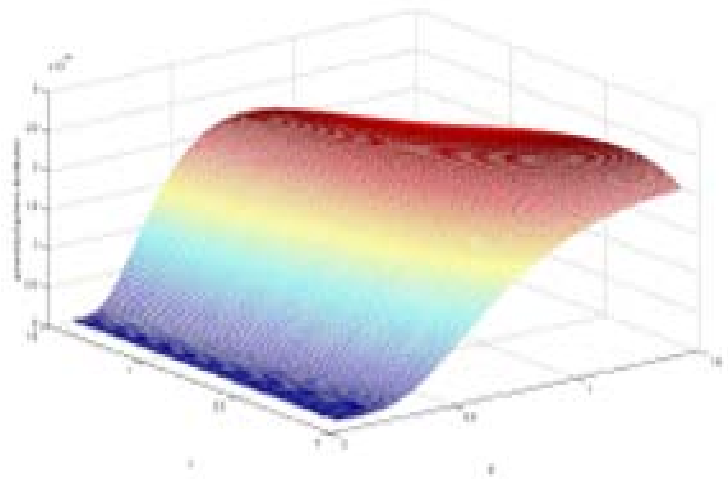

Fig. 5. graph of pdf fanctios venus $p$ with $r=1.4 ; a=1.2 ; m=1.3 ; \lambda=1 ; n=0.1 ; b=1.5$.

Figures 3, 4 \& 5. PDF for different values of $r$ and $\alpha, n, m, p, \lambda, b$, giving a clear idea of the changes caused by the different values of these parameters, those interested can study special cases and note the distinct cases in each change. 
Several structural properties are derived, including explicit expressions for the moments, quantile and generating functions, skewness, kurtosis, mean deviations, Lorenz curves, probability-weighted moments, and two types of entropy. We also investigate the order statistics. The method of maximum likelihood is used for estimating the model parameters and the observed information matrix is derived. We illustrate the flexibility of the new distribution by means of two applications to real datasets. The graph of its pdf and hazard function for various values of the parameters are showed in Figures 6 and 7.

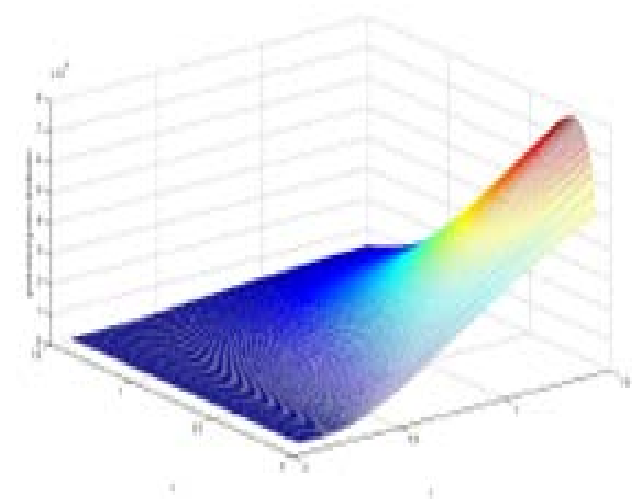

\section{Fig, 6. graph of pdt function venua $f$ with $p=0.01 ; a=1.01 ; \mathrm{m}=0.3 ; \lambda=1.3 ; \mathrm{n}=0.4 ; 6=0.3$.}

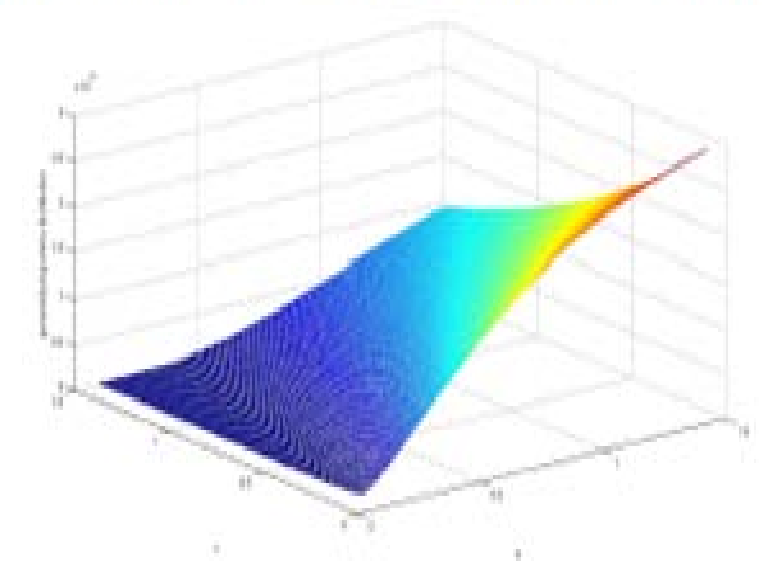

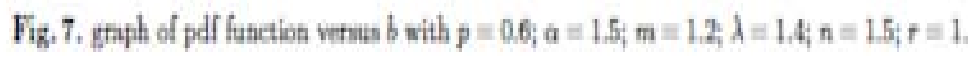

Figures 6 \& 7. PDF for different values of $r$ and $\alpha, n, m, p, \lambda, b$, giving a clear idea of the changes caused by the different values of these parameters, those interested can study special cases and note the distinct cases in each change.

\section{Parameter Estimation for a Extended Generalized Gamma Distribution}

In this paper, we examine a seven-parameter generalization of the extended gamma distribution and derive parameter estimation techniques for that distribution [17]. The difficulties described in the literature made it hard to estimate the parameters of the extended generalized gamma distribution. Those techniques, in the general case, depend upon method of moment's considerations, which lead to simultaneous equations for which closed form solutions are not available. Graphic solution is proposed and aids to the computations are provided $[17,18]$.

\subsection{Moment Estimates}

Let $X_{1}, X_{2}, \ldots, X_{n}$ be a random sample from a random variable $X$ with a pdf as given in (9) which can be rewritten in the following from: 


$$
f(x ; \theta)=\frac{x^{\alpha-1}\left[x^{m}+n\right]^{-r} e^{-\lambda x^{p-1}-\frac{b}{x}}}{\Lambda_{r}(\alpha, n, m, p, \lambda, b)} \quad \text {, where } \theta=(\alpha, n, m, p, \lambda, b, r)
$$

The expression of the $\mathrm{k}^{\text {th }}$ moment about the origin of $X$ can be derived as follows:

$$
\mu_{k}(\theta)=E\left(X^{k}\right)=\int_{0}^{+\infty}\left(x^{k}\right) \frac{x^{\alpha-1}\left[x^{m}+n\right]^{-r} e^{-\lambda x^{p-1}-\frac{b}{x}}}{\Lambda_{r}(\alpha, n, m, p, \lambda, b)} d x=\frac{\Lambda_{r}(\alpha+k, n, m, p, \lambda, b)}{\Lambda_{r}(\alpha, n, m, p, \lambda, b)}
$$

Let $M_{k}$ be the $\mathrm{k}^{\text {th }}$ sample moment, i.e.:

$$
M_{k}=\frac{1}{n} \sum_{i=1}^{n} X_{i}^{k}, \quad k=1,2,3,4,5,6,7 .
$$

Then, the moment estimates are obtained by solving simultaneously each component of $\theta=(\alpha, n, m, p, \lambda, b, r)$ $\theta=(\alpha, n, m, p, \lambda, b, r)$. The following system of seven nonlinear equations $\mu_{k}(\theta)=M_{k}, k=1,2,3,4,5,6,7$. can be solved using Newton-Raphson iterative method [19].

\subsection{Maximum likelihood estimation}

The maximum likelihood estimation (MLE) is a method of estimating the parameters of a statistical model given observations, by finding the parameter values that maximize the likelihood function[17,18]. Thus, the observed data likelihood function for a sample of size $n$ is given by;

$$
\begin{aligned}
L_{n}(x ; \alpha, n, m, p, \lambda, b, r) & =\prod_{i=1}^{n} L_{i}\left(x_{i} ; \alpha, n, m, p, \lambda, b, r\right)=\prod_{i=1}^{n}\left[\frac{x_{i}^{\alpha-1}\left[x_{i}^{m}+n\right]^{-r} e^{-\lambda x_{i} p^{p-1}-\frac{b}{x_{i}}}}{\Lambda_{r}(\alpha, n, m, p, \lambda, b)}\right] \\
= & e^{\sum_{i=1}^{n}\left(-\lambda x_{i}^{p-1}-\frac{b}{x_{i}}\right)} \prod_{i=1}^{n}\left[\frac{x_{i}^{\alpha-1}\left[x_{i}^{m}+n\right]^{-r}}{\Lambda_{r}(\alpha, n, m, p, \lambda, b)}\right]
\end{aligned}
$$

The corresponding log-likelihood function, without the constant term, maximizing the function $L(x ; \theta)=$ lo $\left.\$ L_{n}(x ; \alpha, n, m, p, \lambda, b, r)\right]$ is given by

$L(x ; \theta)=\sum_{i=1}^{n} x_{i}^{\alpha-1}\left[x_{i}^{m}+n\right]^{-r}-\sum_{i=1}^{n}\left(\lambda x_{i}^{p-1}+\frac{b}{x_{i}}\right)-\log \Lambda_{r}(\alpha, n, m, p, \lambda, b) \quad$.The goal of maximum likelihood estimation is to find the values of the model parameters that maximize the likelihood function over the parameter space, that is $L_{n}(x ; \hat{\alpha}, \widehat{n}, \hat{m}, \widehat{p}, \hat{\lambda}, \widehat{b}, \widehat{r})=\operatorname{Sup}_{(\alpha, n, m, p, \lambda, b, r)} L_{n}(x ; \alpha, n, m, p, \lambda, b, r)$. Solving the nonlinear system of equations of $\frac{L(x ; \theta)}{d \alpha}=0$, $\frac{L(x ; \theta)}{d n}=0, \frac{L(x ; \theta)}{d m}=0, \frac{L(x ; \theta)}{d p}=0, \frac{L(x ; \theta)}{d \lambda}=0, \frac{L(x ; \theta)}{d b}=0$ and $\frac{L(x ; \theta)}{d r}=0$ gives the maximum likelihood estimates $\alpha, n, m, p, \lambda, b$ and $r$ respectively. We obtain the $7 \times 7$ observed information matrix through solving the nonlinear system obtained. The solution of the above inverse dispersion matrix yields the asymptotic variance and covariance of the maximum likelihood estimators $\hat{\alpha}, \widehat{n}, \hat{m}, \hat{p}, \hat{\lambda}, \hat{b}, \widehat{r}$. The MLE of $\theta$ say $\alpha, n, m, p, \lambda, b, r$ is obtained by solving the nonlinear system equal zero. This system can be solved using Newton-Raphson iterative method [2]. This nonlinear system of equations does not have a closed form.

\section{Mixture and Fitting of Family of Extended Generalization Gamma Distribution}

The mixture distribution is defined as one of the most important ways to obtain new probability distributions in applied probability and several research areas. According to the previous reason, we have been looking for more flexible alternative to the lifetime data.

Kao (1959) stated that (see also [17]); one has to use mixture of distributions to analyze the data from life-time experiments whenever there are two types of failures such as sudden catastrophic and wear-out failures. Therefore, Radhakrishna et al. [18] suggested using the following pdf of a particular case $n=2$ component mixture generalized gamma 
distributions . The empirical fact confirms that it is difficult to find pure distributions by a full percentage; this makes the researcher use the incomplete claim that the experimental data follows a certain distribution among the known distributions; we expect this expanded generalization of the gamma distribution to allow us to represent many of the mixing models, so we present;

$$
f(x ; \alpha, n, m, p, \lambda, b, r)=\sum_{i=1}^{n} p_{i}\left[f_{i}\left(x ; \alpha_{i}, n_{i}, m_{i}, p_{i}, \lambda_{i}, b_{i}, r_{i}\right)\right], \text { for } 0 \leq p_{i} \leq 1, i=1,2, . . n, \sum_{i=1}^{n} p_{i}=1
$$

Therefore, we introduced a new mixed distribution, namely the mixture extended generalized gamma distribution, which is obtained by mixing between generalized gamma distribution and length based generalized gamma distribution. It is introduced and defined the extended beta distribution and derived analytical shapes of the probability density and hazard rate functions $[10,16]$.

The recent modifications of extended gamma family distribution is capable of modeling bathtub-shaped hazard rate, which contains special sub-models, namely, the exponential, length based exponential, generalized gamma, length based gamma and length based generalized gamma distributions [4, 15].

This new model of extended generalized gamma distribution has more flexibility than other types of extended gamma distributions (three, four, five, and six parameters) due to the shape of its density as well as its hazard rate functions. It was shown that the density of the new distribution could be expressed to have more components: the mixture density function and the generalized gamma density function [8].

We present some useful properties of the extended gamma distribution such as mean, variance, skewness, kurtosis, and hazard rate. Parameter estimations are also implemented using the maximum likelihood method. The application of the extended gamma distribution is illustrated by a real data set [17].

The results demonstrate that recent modifications of extended gamma family distribution can provide the fitted values with more consistent and flexible framework than a number of distributions, which include important lifetime data such as the generalized gamma, length biased generalized gamma, and the five parameters extended Weibull distributions [10]. This generalization of the family of gamma distributions has some renowned sub-models such as the Beta and Weibull while the interval of random variable belongs to fitting distribution generalized exponential distribution, hazard rate function, moment generating function and extensive works on introducing shape parameters for other symmetric distributions which extend the gamma, generalized exponential, Extended Rayleigh, and Weibull with relation to incomplete generalized gamma function [20].

The shapes of the density and hazard rate function with the function of parameters of a gamma distribution for small samples. We describe the extended generalized gamma model and demonstrate how a number of values of parameters covariate to affect the hazard function in a multiplicative manner according to more cases of standard distributions when the shape parameters is equal to small zero values [21].

\section{Reversed Hazard Function}

The reversed hazard function can be obtained using the following relationship:

$$
\mathrm{h},(x, a, n, m, p, \lambda, b)=\frac{f,(x, a, n, m, p, \lambda, b)}{\mathrm{F},(x, a, n, m, p, \lambda, b)}
$$

Therefore, the reversed hazard function of the NEGGD (7) distribution becomes important and allows tracking of many special states formed, whether only from the exponential side or from hybrid states with extended functions, and special cases can be observed in the case of the expanded Weibull distribution or function functions with distributions from generalized families of the distribution. From the same compound formula, can be reviewed the article $[1,17,18]$ for further filing.

\section{Discussion and Application}

The global situation now indicates that the COVID-19 epidemic is spreading everywhere, and the matter has become complicated day by day, and the question has begun when it is possible to end this epidemic, or get the herd's resistance to this epidemic. Tracking the effectiveness of lockdown periods on the spread of the virus will be an important indicator of the success of public health responses around the world. Most governments in the world focus on three indicators-incidence rate, disease severity, and health system capacity - that measure the quality of their response. The chart below shows the paths of the spread of the epidemic in some countries in the world from January to July of 2020 (Days since total confirmed deaths reached 0.1 per million).

The used model NEGGD(7), expressed in equation (9), consists of a three-part structure that allows for the expression of several families of known distributions, and thus rapid and complex exponential random phenomena and their ex- 
tended components can be traced.

Health data modeling and analysis is an important aspect of statistical work in a variety of scientific and medical fields. The new and extended generalized gamma distribution is defined as including seven parameters, which will loosen restrictions on some parameters if the hazard ratio and reliability functions are studied [22].

This family of extended generalized gamma distribution has the monotony characteristic, especially the bathtub for the hazard rate function in terms of the seven parameters, which present important cases when some parameters are fixed.

By the time the curve slopes upward for tracking the spread of the epidemic, the estimation capacity of the new spread map expires, the potential for uncertainty increases, sudden problems in forecasting the expected numbers, and the random characteristic suddenly becomes lost. Generalized gamma model distributions are suitable for modeling data with single-represent risk rates that either increase or decrease the probable patterns, including special cases of distribution such as a generalized exponential distribution or a Weibull spanning when two parameters are fixed, and the extended Relight distribution.

In many cases, the risk rating can resemble a bathtub. The curve initially slopes downward, indicating reduced risk, then stabilizes to remain constant, before moving upwards as the object in question advances.

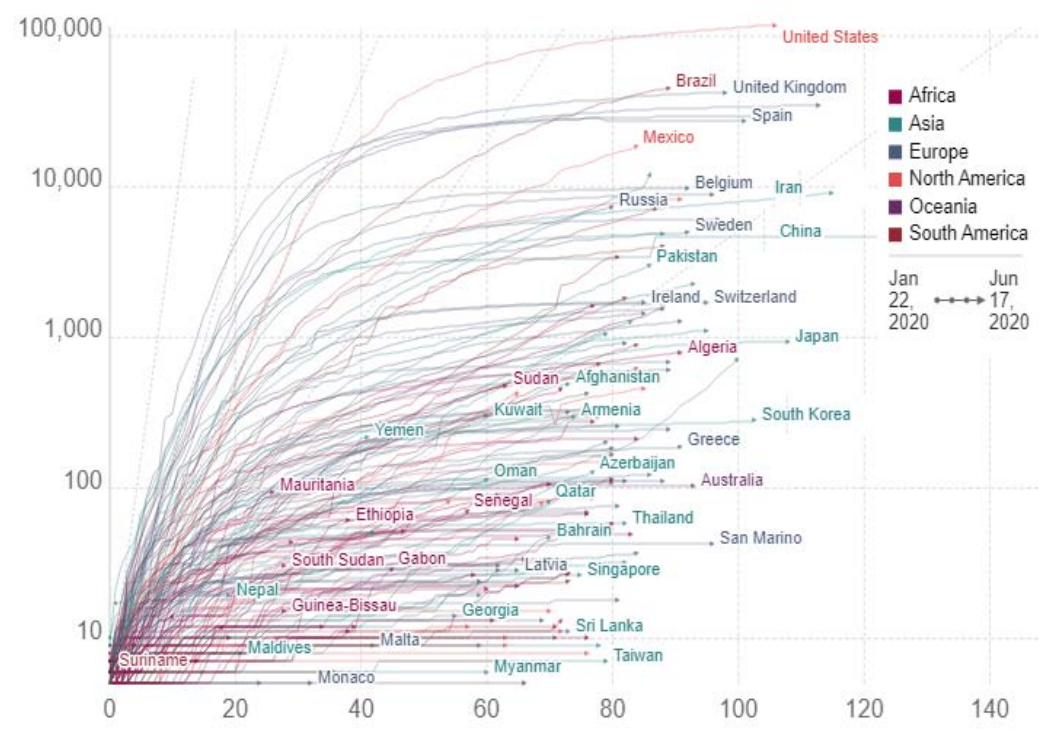

Figure 8. Graphs of the daily number of confirmed cases from January to July to some countries (Source: European Centre for Disease Prevention and Control).

\section{Conclusion}

It is common for analyzes of risk and reliability functions to encounter data and are inconsistent with exponential probability models, Weibull, and other familiar probability models. This data stimulates research to expand the set of probability distributions, which is useful for a reliability analyst. We present and study the model of the extended gamma distribution, which can be interpreted as a truncation of the generalized extended gamma distribution.

\section{References}

[1] Agarwal, S. K. and Kalla, S. L. (1996). A Generalized Gamma Distribution and its Application in Reliability. Commun. Statist. Theory Meth., 25(1), 201-210.

[2] Stacy E. W. and Mihram, G. A., (1965). Parameter Estimation for a Generalized Gamma Distribution. Technimetrics, 7(3), 349-358.

[3] Stacy, E. W. (1962). A Generalization of the Gamma Distribution. Annals of Mathematical Statistics, (33): 1187-1192.

[4] Cordeiro, G. M., Castellares, F., Montenegro, L. C., and de Castro, M. (2012). The Beta Generalized Gamma Distribution. Statistics (Berlin), (46): 1-13.

[5] Satsayamon Suksaengrakcharoen and Winai Bodhisuwan. (2014). A New Family of Generalized Gamma Distribution and Its Application. Journal of Mathematics and Statistics, 10(2): 211-220. 
[6] Bachioua, Lahcene. (2004). On Extended and Reliability General Mixture Gamma Distribution Model. A Dissertation Submitted to The College of Science, University of Baghdad in Partial Fulfillment of The Requirements for The Degree of Doctor of Philosophy (Ph.D.) of Science in Mathematics, University of Baghdad, Iraq.

[7] Bachioua Lahcene, Abdul Moiz Mohammed. (2017). On a Five and Six Parameter Generalization of the Gamma Function. Journal Theoretical Mathematics \& Applications. 7(1): 1-14.

[8] Bachioua, Lahcene. (2013). Extended Generalized Gamma Function and Same its Applications. International Archive of Applied Sciences and Technology, 4(3): 16-30.

[9] Chaudhry M. Aslam and Zubair, S. M. (2001). Extended Gamma and Digamma Functions. Fractional Calculus and Applied Analysis, 4(3): 303-326.

[10] Bachioua, Lahcene. (2018). On Recent Modifications of Extended Weibull Families Distributions and its Applications. Asian Journal of Fuzzy and Applied Mathematics, 6(1): 1-11.

[11] Kobayashi, K. (1991). On a Generalized Gamma Function Occurring in Diffraction Theory. Journal of Physical Society of Japan, (60), 1501-1512.

[12] Abdul Moiz Mohammed, Bachioua Lahcene. (2017). On Extension of Euler’s Beta Function. Journal of Applied Mathematics \& Bioinformatics, Scienpress Ltd., 7(3): 1-11.

[13] Chaudhry M. Aslam, Asghar Qadira, Rafique, M., and Zubair, S. M. (1997). Extension of Euler’s beta function. Journal of Computational and Applied Mathematics, (78): 19-32.

[14] Amoroso, L. R., (1925). Around the Income Curve. Annals of Mathematica, Series 2(1), 123-159.

[15] Johnson, N. L., Kotz, S., and Balakrishnan, N. (1994). Continuous Univariate Distributions. Vol. 1, 2nd edition, Wiley and Sons, Inc., New York.

[16] Jones, M. (2015). On Families of Distributions with Shape Parameters. International Statistical Review, (83), 175-192.

[17] Falls, L. W. (1970). Estimation of Parameters in Compound Weibull Distributions. Technimetrics, 12(2), 339-407.

[18] Radhakrishna, C., et al. (1992). Estimation of Parameters in a Two Component Mixture Generalized Gamma Distribution. Commun. Stat. theory Meth, 21(6): 1799-1805.

[19] Stacy, E. W. and Mihram, G. A. (1965). Parameter Estimation for a Generalized Gamma Distribution. Technimetrics, 7(3), 349-358.

[20] Bachioua, Lahcene. (2017). On Recent Modifications of Extended Rayleigh Distribution and its Applications. JP Journal of Fundamental and Applied Statistics, 11(1): 1-13.

[21] Bachioua, Lahcene. (2018). On Extended Normal Distribution Model with Application in Health Care. International Journal of Statistics in Medical Research, 7(3): 88-95.

[22] Bachioua, Lahcene. (2020). On Extended Exponential Distribution: Properties and Applications In Tracking the Pandemic Covid-19. Journal of SunText Review of Medical and Clinical Research, Open Access Research Article, SunText Rev Med Clin Res., 1(2): 109. 